\title{
Schlechtes Omen bei diabetischem Fuß
}

Fragestellungen: Ist für die hohe Mortalität von Patienten mit diabetischem Fußsyndrom auch die Diabeteseinstellung und eine Verlängerung der herzfrequenzkorrigierten QT-Zeit (QTc) ursächlich, und falls ja wie sehr?

Hintergrund: Patienten mit diabetischem Fuß, vor allem jene mit pAVK, haben eine sehr hohe - v.a. kardiovaskuläre - Mortalität. Einige prospektive Langzeitstudien ergaben eine geringere kardiovaskuläre Mortalität bei guter Diabeteseinstellung. In der ACCORD-Studie aber wurde eine höhere Mortalität bei intensiver Diabetestherapie und niedrigen $\mathrm{HbA}_{1 c}$-Werten beobachtet. Die Bedeutung von Hypoglykämien für die Mortalität bei Typ-2-Diabetikern wird seither intensiv diskutiert. Eine verlängerte QTc-Zeit ist mit erhöhter Mortalität assoziiert. Patienten mit diabetischer kardialer autonomer Neuropathie und jene mit Hypoglykämien sind davon vermehrt betroffen.

Patienten und Methodik: Alle Patienten, die sich binnen zwei Jahren mit diabetischem Fußulkus in der Fußambulanz von Lund vorstellten, wurden gescreent. Wenn ihr Ulkus nach vier Wochen nicht abgeheilt war, wurden sie in die Studie aufgenommen $(n=224)$. Dialysepflichtige wurden nicht berücksichtigt, auch Teilnehmer mit fehlenden $\mathrm{HbA}_{1 \mathrm{c}}$-Werten nicht. Die QTcZeit wurde mithilfe eines automatischen Analyse-Programms (Megacart, Siemens) bestimmt und auf die Herzfrequenz mit der Bazett-Formel korrigiert. Eine QTc-Zeit größer als $440 \mathrm{~ms}$ wurde als pathologisch betrachtet. Die Patienten kamen je nach $\mathrm{HbA}_{1 \mathrm{c}}$ in eine von drei Gruppen: $<7,5 \%, 7,5-8,9 \%,>8,9 \%$. Das mediane Alter betrug 69 Jah-

Fagher K, Löndahl M. The impact of metabolic control and QTc prolongation on all-cause mortality in patients with type 2 diabetes and foot ulcers. Diabetologia 2013;56:1140-7. re (in allen Gruppen ähnlich), 62\% waren Männer. 81 befanden sich in der Gruppe $1\left(\mathrm{HbA}_{1 \mathrm{c}}<7,5 \%\right), 70$ in 2 $\left(\mathrm{HbA}_{1 \mathrm{c}} 7,5-8,9 \%\right)$ und 63 in 3 $\left(\mathrm{HbA}_{1 \mathrm{c}}>8,9 \%\right)$. Es gab mehr Patienten mit pAVK und ver- längerter QTcZeit in der Gruppe mit $\mathrm{HbA}_{1 \mathrm{c}}$ $<7,5 \%$. Medikamente, die die QTc-Zeit verlängern können, waren in allen Gruppen gleich verteilt.

Ergebnisse: In der Nachbeobachtungszeit von 8 Jahren starben 151 Patienten (70,6\%). Ein $\mathrm{HbA}_{1 \mathrm{c}}<7,5 \%$ war signifikant mit einer erhöhten Mortalität assoziiert. In einem Cox-Hazard-Modell wurden verschiedene Risikofaktoren berücksichtigt. Nur Alter, Geschlecht, $\mathrm{HbA}_{1 \mathrm{c}}$ und verlängerte QTc-Zeit beeinflussten signifikant die Mortalität. Ein $\mathrm{HbA}_{1 \mathrm{c}}<7,5 \%$ und eine Verlängerung der QTc-Zeit verursachten unabhängig voneinander eine höhere Mortalität. Eine Subgruppenanalyse zeigte die höchste Sterblichkeit für Patienten mit $\mathrm{HbA}_{1 \mathrm{c}}<7,5 \%$ und Verlängerung der QTc-Zeit. 92\% dieser Patienten waren nach 8 Jahren gestorben, aber nur $49 \%$ derer mit einem $\mathrm{HbA}_{1 \mathrm{c}}<7,5 \%$ und normaler QTc-Zeit.

Schlussfolgerungen: Patienten mit verlängerter QTc-Zeit haben eine besonders hohe Mortalität, falls ihr $\mathrm{HbA}_{1 c}<7,5 \%$ liegt. Als mögliche Ursache werden Hypoglykämien mit folgenden Herzrhythmusstörungen diskutiert. Daher sollten Patienten mit diabetischem Fußsyndrom ein EKG-Screening erhalten. Bei verlängerter QTc-Zeit sollte man Medikamente vermeiden, die die QT-Zeit negativ beeinflussen, der $\mathrm{HbA}_{1 \mathrm{c}}$ sollte nicht $<7,5 \%$ sein, und Hypoglykämien müssen vermieden werden.

\section{- Kommentar von Prof. Dr. med. Maximilian Spraul}

\section{Unterzuckerungen unbedingt vermeiden!}

Die Studie macht an einem relativ kleinen Kollektiv mit diabetischem Fußulkus die erhebliche Mortalität dieser Patienten deutlich. Eine verlängerte QTc-Zeit und ein niedrigerer $\mathrm{HbA}_{1 \mathrm{c}}$ wiesen auf eine erhöhte Mortalität hin. Die Ergebnisse deuten an, dass bei diesen Patienten mit schweren Komorbiditäten ( $p A V K, K H K$, Neuropathie) eine gute Betreuung und effektive Therapie wichtiger ist als eine normnahe Blutzuckereinstellung. Hier muss man der Diagnose einer verlängerten QTc-Zeit vielleicht deutlich mehr Aufmerksamkeit widmen als bisher üblich. Hypoglykämien sollten bei den meist multimorbiden Patienten mit diabetischem Fußsyndrom vermieden werden.

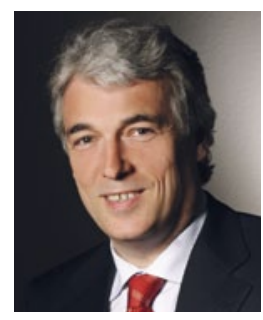

Prof. Dr. med. Maximilian Spraul

Mathias-Spital \& Jakobi-Krankenhaus (Med Klinik III),

Interdisziplinäres Diabetes-Fuß-Zentrum

Frankenburgstr. 31

48431 Rheine

m.spraul@mathias-spital.de 